\title{
DESENHO DE EXPERIÊNCIAS COLABORATIVAS PARA A EDUCAÇÃO A DISTÂNCIA
}

\author{
Ana-Paula Correia | acorreia@iastate.edu \\ $\mathrm{PhD}$ em Instructional Systems Technology pela Indiana University, USA. Professora do Programa de Pós-graduação a Distância em \\ Curriculum and Instructional Technology da Iowa State University, EUA.
}

\section{Resumo}

Este artigo apresenta um estudo de caso contextualizado no Programa de Pós-graduação a Distância em Curriculum e Instructional Technology da Iowa State University EUA. Este programa foi criado para servir professores do K-12 (pré-primária até à décima-segunda série) que, sem ele não teriam a oportunidade de completar uma pós-graduação em Educação.

O modelo de aprendizagem experiencial de David Kolb é usado para estruturar as atividades de aprendizagem colaborativa e usado como quadro de referência para o desenvolvimento de um dos cursos a distância em Desenho Educacional. Este curso é discutido em detalhe no que respeita à comunidade de aprendizagem formada, abordagens de ensino utilizadas e projetos desenvolvidos com a finalidade de resolver problemas educacionais reais. Exemplos desses projetos realizados por equipes virtuais são fornecidos ao mesmo tempo que a sua pertinência em termos práticos é explicada. De igual forma, a avaliação dos alunos e do curso é discutida. Este artigo termina com sugestões para o desenvolvimento de cursos a distância que usem um quadro de referência idêntico como forma de contribuir para uma reflexão que potencie um uso mais alargado de aprendizagem experiencial e colaborativa na Educação a Distância.

\section{Palavras-chave}

Aprendizagem experiencial. Aprendizagem colaborativa. Desenho educacional. 


\section{Abstract}

This paper introduces a case study that took place in the context of the Iowa State University at-a-distance Master in Curriculum \& Instructional Technology in the United States. This program was created to serve K-12 teachers that without it would not have the chance to pursue an advanced degree in Education.

David Kolb's experiential learning is used as a framework to create a graduate education course in instructional design and to design collaborative learning activities. The learning community established, approaches to teaching, and class projects grounded on real-world instructional problems are discussed in detail and all part of this course. Examples of such virtual group projects are provided as well as an explanation of these projects' relevance for practice. At the same time, student and course evaluation are discussed. This article ends with recommendations for the development of at-a-distance courses that use similar theoretical frameworks as a way to contribute to the discussion on bringing experiential and collaborative learning to Distance Education.

\section{Keywords}

Experiential Learning. Collaborative learning. Instructional design.

\section{Resumen}

Este artículo presenta un caso de estudio contextulaizado en el Programa de Postgrado a distancia en Currículo y Tecnología Educativa en la Iowa State University, Estados Unidos. Este programa fue creado para servir maestros de K-12 (pre-primaria hasta el duodécimo grado) que, sin este programa no tendría la oportunidad de terminar un postgrado en Educación.

El modelo de aprendizaje experiencial de David Kolb se utiliza para estructurar las actividades de aprendizaje colaborativo y como marco de referencia para el desarrollo de un curso a distancia en el diseño educativo. Este curso se discute en detalle con respecto a la comunidad de aprendizaje formada, los enfoques pedagógicos utilizados y desarrollo del proyectos con el propósito de resolver problemas reales de la educación. Se proporcionan ejemplos estos proyectos lleva- dos a cabo por los equipos virtuales al mismo tiempo que se explica su importancia práctica. Asimismo se discute la evaluación de los estudiantes y el curso. Este artículo termina con algunas sugerencias para el desarrollo de cursos a distancia.

\section{Palavras-clave}

Aprendizaje experimental. Aprendizaje de colaboración. Diseño educacional..

\section{Introdução}

No contexto dos Estados Unidos, muitos são os programas de pós-graduação que se focam mais na transmissão e replicação do conhecimento do que no proporcionar de autênticas oportunidades de ensinoaprendizagem e/ou no "[testar] em ação aquilo que o aluno aprende" (KOLB; KOLB, 2005, p. 208; tradução nossa). Essa situação é ainda mais gritante nos muitos programas de ensino a distância. Estes transmitem enormes quantidades de informação em vez de oferecer pedagogias e processos que permitem o desenvolvimento de competências transferíveis para os contextos de trabalho (MCLOUGHLIN; LUCA, 2002). Essa tendência pedagógica de privilegiar a transmissão de conhecimento em detrimento de experiências autênticas é problemática. $\mathrm{O}$ mundo de trabalho espera das universidades que estas preparem profissionais capazes de usar as TIC em contextos educativos para resolver problemas complexos $e$ desempenhar atividades que requerem um elevado nível de especialização. Este objetivo será difícil de alcançar se cursos a distância não oferecerem oportunidades para os alunos porem em prática aquilo que aprendem e experienciarem os problemas e benefícios de lidar com situações autênticas - situações que vão para além da análise de estudos de caso e/ ou simulações computadorizadas.

Este artigo apresenta um estudo de caso em que alunos de pós-graduação inscritos num curso a distância em Desenho Educacional aplicam o conhecimento e competências desenvolvidas lidando com autênticos problemas educativos. O modelo de aprendizagem 
experiencial de David Kolb é usado para estruturar as atividades de aprendizagem colaborativa e como quadro de referência para o desenvolvimento deste curso a distância.

\section{Enquadramento teórico}

\section{1 modelo de Kolb para aprendizagem experiencial}

O modelo de Kolb para aprendizagem experiencial serviu de base para o desenho das atividades deste curso. Aprendizagem experiencial é um processo de construção do conhecimento que envolve "uma tensão criativa entre quatro formatos de aprendizagem" (KOLB; KOLB 2005, p. 194; tradução nossa). Os quatro formatos de aprendizagem - experiência concreta ("concrete experience"), observação reflectiva ("reflective observation"), conceitualização abstrata ("abstract conceptualization"), e experimentação activa ("ative experimentation") - estão organizados num padrão cíclico conhecido como ciclo de aprendizagem experiencial (Figura 1).

No contexto deste estudo, as experiências concretas resultaram da participação dos alunos, eles próprios professores do ensino primário e secundário ou pessoas ligadas à educação. A conceitualização abstrata tomou forma através das múltiplas discussões assíncronas e síncronas fomentadas no curso. As discussões assíncronas foram estruturadas segundo três diferentes

Figura 1: Ciclo de aprendizagem experiencial (KOLB, 1984).

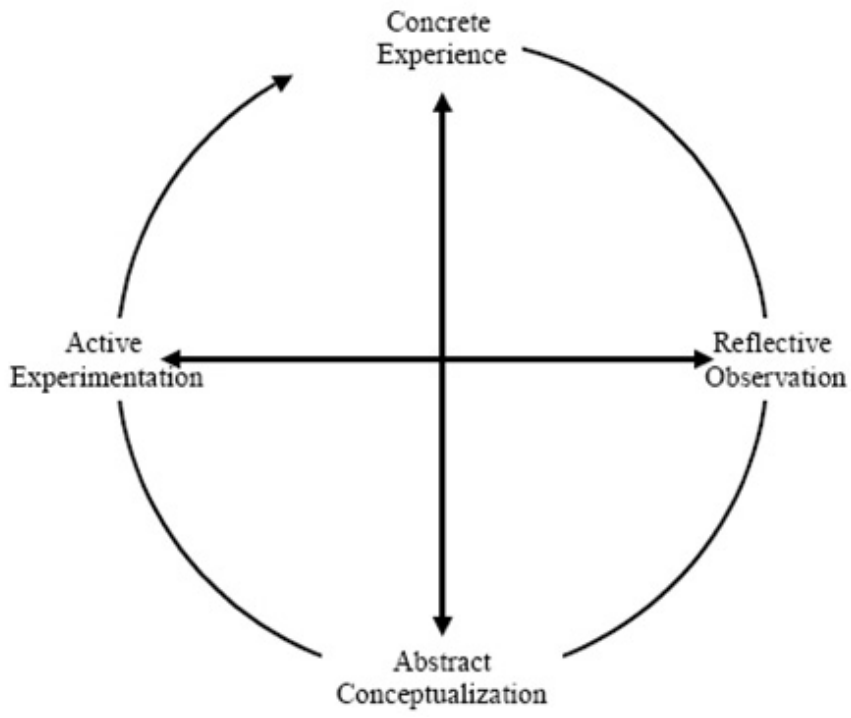

formatos: (1) todos os alunos participavam ao mesmo tempo nas discussões facilitadas pela docente do curso; (2) os alunos organizados em pequenos grupos (6 a 8 alunos cada) participavam nas discussões facilitadas pela docente; e (3) os alunos organizados em pequenos grupos participavam nas discussões facilitadas por eles próprios (cada semana um ou dois alunos ofereciam-se como voluntários para liderar a discussão) (CORREIA; DAVIS, 2007).

Adicionalmente a conceitualização abstrata tomou também forma através das numerosas leituras realizadas pelos alunos e problematizadas pela docente. Princípios de desenho educacional, estratégias e modelos para o desenho educacional e aplicações práticas destes conceitos teóricos foram muitos dos tópicos abordados nas discussões síncronas via chat.

Notas e diários reflectivos baseados na experiência e atividade profissional dos alunos e na sua participação neste curso constituíram a observação reflectiva proposta no ciclo de aprendizagem experiencial. A experimentação ativa consistiu no culminar das atividades do curso a distância. Os alunos foram incentivados a aplicar os conhecimentos e competências que iam aprendendo no curso na resolução de problemas educacionais reais. Enquanto as atividades de aprendizagem no curso a distância foram planeadas de acordo com o ciclo de aprendizagem experiencial, este artigo centra-se no último quadrante do ciclo, experimentação ativa (Figura 2).

Figura 2: Modelo de Kolb e atividades de aprendizagem do curso a distância.

\begin{tabular}{|l|l|}
\hline $\begin{array}{l}\text { Experiência } \\
\text { concreta }\end{array}$ & $\begin{array}{l}\text { Observação } \\
\text { reflectiva }\end{array}$ \\
$\begin{array}{l}\text { Discussões } \\
\text { assíncronas } \\
\text { estruturadas }\end{array}$ & $\begin{array}{l}\text { Notas e diários } \\
\text { reflectivos }\end{array}$ \\
\hline $\begin{array}{l}\text { Experimentação } \\
\text { ativa } \\
\text { Produtos } \\
\text { educacionais de } \\
\text { aplicação real }\end{array}$ & $\begin{array}{l}\text { Conceitualização } \\
\text { Abstrata } \\
\text { Discussões } \\
\text { assíncronas e } \\
\text { síncronas }\end{array}$ \\
\hline
\end{tabular}


Este artigo descreve a atividade mais importante do curso: a criação de um grupo de produtos educacionais desenhados para serem usados em situações autênticas. Esses produtos eram desenhados para servir um grupo particular de aprendentes e serem usados num contexto específico (CORREIA, 2008). Exemplos dos projetos desenvolvidos neste curso serão descritos aquando da apresentação do estudo de caso.

\subsection{A aprendizagem colaborativa}

O conceito de aprendizagem colaborativa é polissémico, pois varia conforme os contextos em que é utilizado. No contexto deste artigo, aprendizagem colaborativa representa o esforço intelectual desenvolvido conjuntamente pelos alunos ou pelos alunos $e$ professores (SMITH; MACGREGOR, 1992). Num cenário típico, os alunos trabalham em equipes de dois a quatro elementos, explorando conceitos, desenvolvendo entendimentos mútuos e buscando soluções ou significados para os desafios propostos.

Grande parte da investigação relacionada com equipes de trabalho a distância enquadra-se no âmbito das comunidades de aprendizagem. Na maioria dos casos, as comunidades de aprendizagem são definidas em termos de construção de conhecimento, inspiradas nas teorias de aprendizagem construtivista ou socioconstrutivista. A construção de conhecimento ocorre durante a realização de projetos que valorizam a negociação de significados e evolvem da colaboração $e$ participação na comunidade de aprendizagem. Estas relações destacam a importância da colaboração para a aprendizagem em rede. Como Harasim (2000) explica: "Os princípios relacionadas com a aprendizagem colaborativa parecem ser o conceito mais importante para o ensino-aprendizagem a distância, uma vez que estes princípios apoiam fortemente a aprendizagem socioafectiva e cognitiva que ocorre na Web" (p. 53; tradução nossa).

A aprendizagem colaborativa exige por parte do professor menos controle, um enquadramento flexível e uma maior autonomia do aluno, num clima de responsabilidade e autonomia. O trabalho colaborativo não é simplesmente a soma ou justaposição de trabalhos individuais, mas mais do que isso, exige: (1) um tremendo envolvimento individual focalizado no bem- próprio do grupo como entidade autónoma, (2) uma formulação de objetivos comuns e (3) uma constante coordenação de atividades. $\mathrm{O}$ envolvimento tem que ir para além da simples interacção, pois como acentua Murphy (2004), a interação com os outros pode ser o ponto de partida da colaboração, mas para existir uma colaboração autêntica é necessário algo mais do que a simples interação entre pares. Segundo Levan (2004), o trabalho colaborativo define uma modalidade de ação que ultrapassa a ação individual e inscreve-se também, explicitamente, numa dinâmica de ação coletiva. É, assim, um processo social que requer o envolvimento de todos os membros da equipe. Nessa mesma linha esse autor explica que a motivação, a comunicação e a interação estão no cerne de um trabalho colaborativo eficaz (LEVAN, 2004). Assim, a colaboração requer a "aceitação dos outros, no respeito, mas também na partilha de autoridade, consenso e ausência de competição. Na colaboração, a realização da tarefa articula-se mais num envolvimento pessoal, mas num ambiente de interação que possibilita a entreajuda mútua $e$ a pôr em comum o fruto do seu trabalho. O grupo, sem ser o único local de aprendizagem, é um local privilegiado de troca de informações e saberes" (MEIRINHOS, 2006, p. 122).

A abordagem de ensino usada no curso a distância em Desenho Educacional baseia-se fortemente nos princípios e práticas da aprendizagem colaborativa. As próximas seções deste artigo descrevem os aspectos pedagógicos do curso como a criação de uma comunidade de aprendizagem, a abordagem de ensino usada, os projetos desenvolvidos em equipes virtuais $e$ a avaliação dos alunos e do curso.

\section{Um estudo de caso: curso de pós-graduação em Desenho Educacional}

Fundamentos de Desenho Educacional é um curso de pós-graduação incluído no Programa de Pósgraduação a Distância em Curriculum \& Instructional Technology da Iowa State University, EUA. Este programa foi criado para servir professores do K-12 (préprimária até à décima-segunda série) distribuídos pelas escolas rurais do estado de lowa. Se não fosse esse programa, esses professores não teriam a oportunidade de completar uma pós-graduação em Educação. 
O estado de Iowa situa-se no centro dos Estados Unidos, sendo a agricultura e a criação de suínos as suas maiores fontes de rendimento. Os professores que ensinam nas áreas rurais sentem-se geralmente isolados em relação aos seus colegas e limitados em termos de oportunidades de progresso na carreira.

O Programa de Pós-graduação a Distância em Curriculum \& Instructional Technology consiste em dez cursos que perfazem um total de 32 unidades de crédito educacional. Os cursos a frequentar exploram não só questões de Desenho Educacional, mas também outras questões como, por exemplo, fundamentos de tecnologia educativa, integração das TIC no ensinoaprendizagem, multiculturalismo no ensino a distância, metodologias de investigação em ação, teorias curriculares e história da educação no contexto dos Estados Unidos. O diploma é conferido após a submissão de um portfólio e passagem num exame oral liderado por três docentes do programa.

\subsection{A comunidade de aprendizagem}

O curso a distância em Desenho Educacional tinha 17 alunos inscritos, sendo quatro do sexo masculino e 13 do sexo feminino, com idades compreendidas entre 22 e 45 anos. Destes alunos, oito faziam parte do primeiro grupo de alunos inscritos no programa de pós-graduação a distância. A maioria destes eram professores de História, Inglês, Arte e Computadores em escolas do Ensino Primário e Secundário distribuídas por todo o estado de Iowa. Os nove restantes eram alunos de pós-graduação empregados pela Iowa State University em diferentes capacidades como, por exemplo, coordenadores educacionais, assistentes em cursos de tecnologia educativa e especialistas em ensino a distância. Este último grupo de alunos não tinha muita experiência de ensino nos contextos de $\mathrm{K}-12$, e uma vez que trabalhavam no campus universitário tinham um acesso privilegiado à docente do curso. Esta dicotomia entre esses dois grupos de alunos pertencentes à mesma comunidade de aprendizagem, mas com experiências e objetivos profissionais muito diferentes causou um certo conflito e fricção, de alguma forma difícil de gerir, dada a natureza a distância do curso (CORREIA; DAVIS, 2008).

A maioria das atividades do curso, incluindo as atividades em equipe ocorreu em WebCT, um produto da Blackboard Inc. Adicionalmente, os alunos tinham também de participar em duas sessões presenciais no campus universitário, o que exigia que se deslocassem fisicamente à universidade. $\mathrm{O}$ objetivo desses encontros era fomentar o desenvolvimento de uma comunidade de aprendizagem ativa e coesiva. Essas sessões eram especialmente apreciadas pelos alunos, uma vez que estes tinham a oportunidade de interagir com os colegas e a docente do curso. Na maioria dos casos, os outros docentes do programa e a coordenadora participavam do almoço com os alunos. Essas reuniões sociais tinham a finalidade de estreitar as relações profissionais entre todos os participantes e apoiar o sucesso do programa de pós-graduação.

\subsection{Abordagens de ensino}

Duas abordagens de ensino guiaram a docente no desenho, desenvolvimento e implementação do curso a distância em Desenho Educacional:

1. Aprender em situações autênticas. Indivíduos aprendem melhor quando ativamente envolvidos em atividades de aprendizagem diretamente relacionadas com os seus interesses e necessidades. Grande parte da aprendizagem neste curso ocorreu no âmbito de projetos e situações similares àquelas que os alunos iriam encontrar e experienciar em situações profissionais depois de terminar a pós-graduação.

2. Aprender como membro de uma equipe virtual. Como complemento à aprendizagem dos conteúdos do curso os alunos também aprendem conceitos, métodos e técnicas necessárias para se tornarem excelentes membros de equipes virtuais. Nesse sentido, os alunos do curso eram não só membros de uma comunidade de aprendizagem alargada, mas também membros de equipes virtuais envolvidas em resolver problemas educacionais.

A docente deste curso esforçou-se também em estabelecer uma estreita parceria com os seus alunos através de uma série de atividades, tais como: (1) constante solicitar de feedback aos alunos sobre o curso, à medida que este se desenrolava para que as atividades de aprendizagem fossem desenvolvidas de acordo com os seus interesses e aspirações, e (2) contínuo incentivo dos alunos para que desenvolvessem projectos autênticos com um for- 
te vínculo às suas atuais actividades profissionais. Dessa forma, os alunos poderiam rentabilizar ao máximo o curso, usando os projetos de Desenho Educacional como uma estratégia para enriquecer suas vidas profissionais.

\subsection{Projetos de desenho educacional desenvolvidos pelos alunos como membros das equipes virtuais}

A atividade de aprendizagem mais importante do curso foi o desenvolvimento de um projeto educacional com o objetivo de educar um grupo particular de aprendentes num tópico e para um contexto específico. Os alunos trabalharam em equipes virtuais para criar diferentes experiências educacionais. A finalidade destas experiências era contribuir para solucionar um problema educacional identificado nas comunidades onde os alunos viviam ou trabalhavam. Neste sentido, os alunos eram incentivados para que o projeto estivesse de algum modo ligado a um problema social sentido por um grupo de aprendentes desfavorecidos. Consequentemente, as equipes tiveram não só que escolher o conteúdo a ensinar, mas também de identificar um grupo de aprendentes e um contexto de aprendizagem real. As seguintes atividades foram requeridas a todas as equipes:
- seleção do tópico a ensinar;

- seleção do grupo de aprendentes e contextos de aprendizagem;

- levantamento de necessidades referentes aos aprendentes e contextos;

- justificação da experiência educacional a desenhar;

- desenvolvimento das especificações para o desenho educacional;

- criação dos materiais educacionais;

- implementação da experiência educacional;

- avaliação formativa da experiência educacional.

Equipe "Cobra". Esta equipe desenvolveu um portal online desenhado para apoiar alunos e professores internacionais trabalhando na Iowa State University a se adaptarem culturalmente às exigências de uma nova cultura acadêmica, estilo de trabalho e modo de vida nos Estados Unidos. Adicionalmente, esse portal incluía recursos de apoio a indivíduos e grupos que viviam longe do seus países de origem, famílias e amigos próximos. Tópicos como, por exemplo, o ajuste e choque cultural, diferenças e estereótipos culturais fizeram parte desta experiência educacional.

Figura 3: Imagem extraída do «International Community Resources», portal online

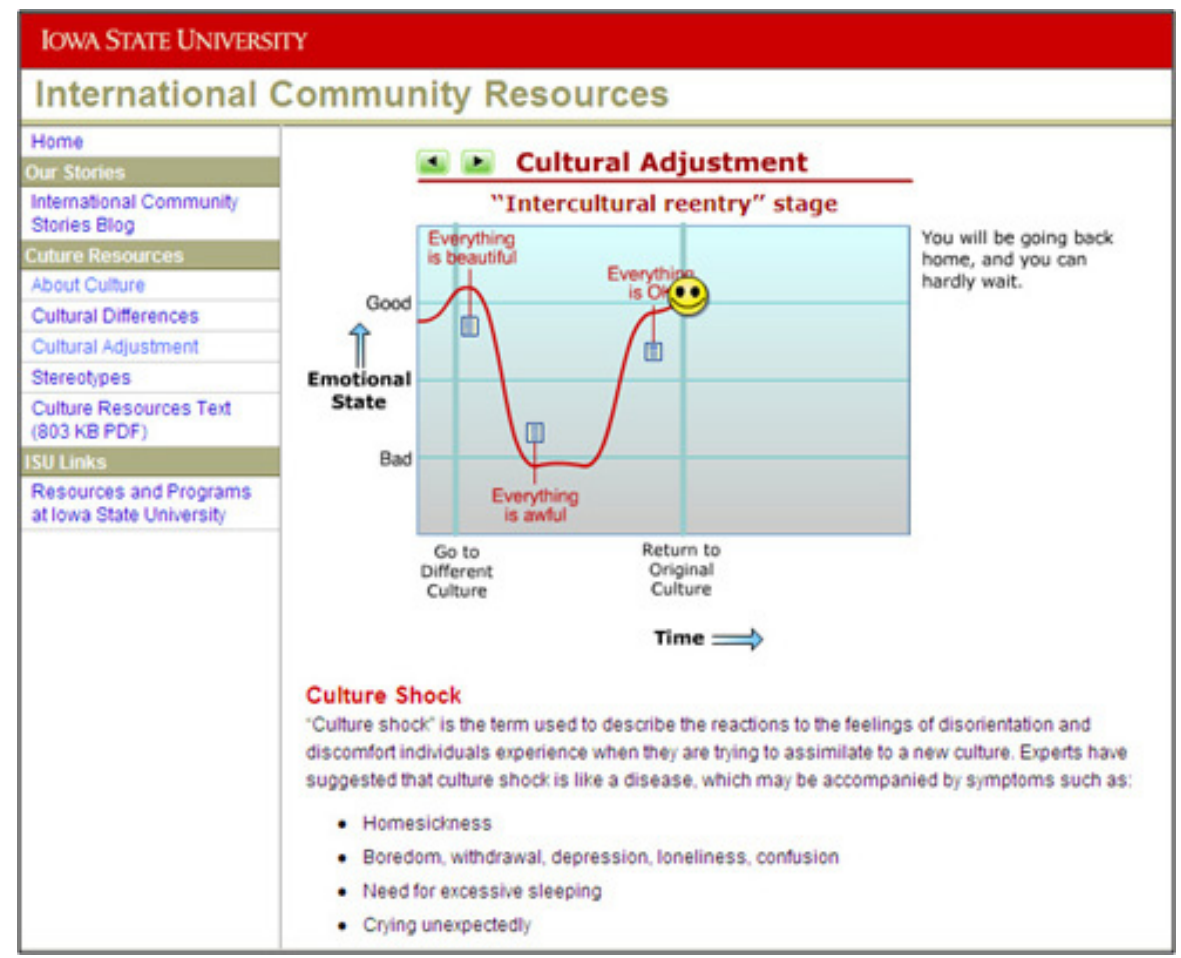


Equipe "Urso". O projeto desenvolvido por esta equipe abordou o ensino da Matemática para crianças dos 3 aos 5 anos. Um workshop foi desenhado para os pais dessas crianças, com o objetivo de os equipar com estratégias de ensino de simples conceitos matemáticos embebidos em atividades cotidianas. A equipe criou uma série de atividades diferenciadas para incentivar mesmo aqueles pais que não se sentiam muito à vontade com a Matemática. Como exemplo, um calendário com sugestões para diferentes atividades (por exemplo, quando os pais e as crianças chegam a casa do supermercado convidar as crianças a contar o número de itens contidos nos sacos de compras) foi distribuído a todos os participantes do workshop.
Equipe "Águia". O tópico que esta equipe abordou relacionou-se com a preparação necessária para enfrentar os rigorosos e prolongados invernos em Iowa, e para tal desenvolveu uma unidade didáctica para a web. Os alunos da universidade foram os grupos de aprendentes alvo para esta unidade, pois muitos deles não sabiam o que fazer para lidar com temperaturas entre os -6 e -20 graus durante os meses de novembro a fevereiro. Importante lições sobre o apropriado vestuário a usar, como conduzir e caminhar em segurança na neve e no gelo e como evitar situações perigosas como hipotermia e congelamento das extremidades foram incluídos nesta unidade.

Figura 4: Imagem extraída da uma unidade didática para a web sobre como se preparar para o inverno rigoroso de Iowa, nos Estados Unidos.

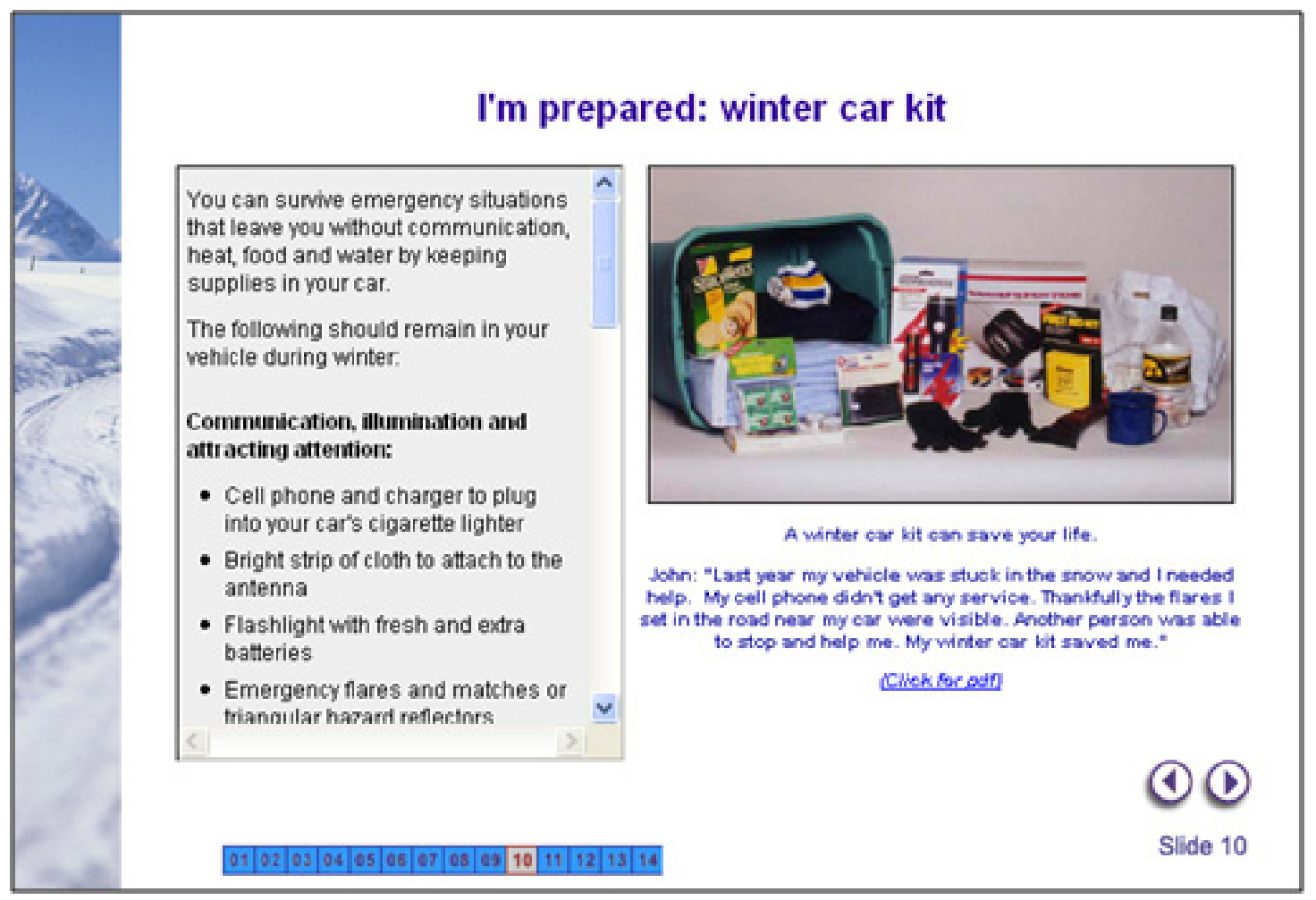

\subsection{Avaliação dos alunos}

Os projetos de desenho educacional foram avaliados em relação aos processos de desenho utilizados e produtos educacionais criados. Esses projetos incluíram vários trabalhos intermediários, tais como: (1) um relatório sobre o levantamento de necessidades referente aos aprendentes e contextos, (2) uma explicação das especificações para o desenho educacional e (3) uma série de desenho iniciais dos produtos educacionais. Esses relatórios intermediários permitiram aos alunos documentar e refletir sobre o processo de desenho educacional e criar um projeto solidamente fundamentado nos conhecimentos e competências desenvolvidas no curso.

Os critérios de avaliação usados para classificar cada um desses relatórios e o produto final foram cuidadosamente comunicados em detalhe aos alunos para evitar surpresas e mal-entendidos quando da saída das classificações. Adicionalmente, a docente ofereceu contínuo feedback aos alunos sobre o 
seu desempenho durante todos os passos do projeto, pelo que os alunos tiveram a oportunidade de incorporar as suas frequentes sugestões à medida que o projeto progredia.

Numa das sessões presenciais realizadas no campus da lowa State University cada equipe teve que apresentar durante 30 minutos os materiais educacionais desenvolvidos assim como descrever a natureza, audiência e contextos de prática relativos aos seus projetos. As equipes foram avaliadas de acordo com a qualidade educacional dos produtos criados e o rigor do processo de desenho educacional seguido. Essa avaliação foi baseada não só nos critérios de qualidade estabelecidos pela docente, mas também nos comentários e críticas feitas pelos respectivos colegas.

Adicionalmente, no fim do projeto os alunos tiveram que submeter uma avaliação de desempenho relativa a cada membro da sua equipe. Os alunos eram avaliados pelos seus colegas de equipe em três dimensões: participação nas tarefas do projeto, capacidade de partilha e dependabilidade. Essa avaliação também incluía uma autoavaliação na qual cada aluno descrevia em detalhe a sua participação no projeto.

A classificação dos projetos de desenho educacional desenvolvidos pelas equipes virtuais dependeu da soma dos seguintes aspectos: (1) qualidade educacional dos materiais criados $(45 \%$ da classificação final no projeto); (2) extensão e detalhe dos trabalhos intermediário (60\%); (3) rigor do relatório final (25\%); e (4) autoavaliação e avaliação dos membros da equipe (10\%).

Uma reflexão crítica sobre o curso foi também requerida aos alunos. Este exercício individual exigia que cada aluno refletisse sobre a sua aprendizagem no curso em termos dos ganhos pessoais e profissionais e representou uma oportunidade adicional para os alunos se pronunciarem sobre a qualidade das experiências educacionais oferecidas durante o curso.

\subsection{Avaliação do curso}

Uma análise das reflexões partilhadas pelos alunos mostra que estes foram unânimes em afirmarem o muito que aprenderam no curso a distância em Desenho Educacional. Muitos deles referiram não só terem aprendido sobre modelos e teorias de de- senho educacional, mas igualmente sobre como se tornar um excelente membro de uma equipe virtual. Entretanto, a complexidade dos projetos de equipe e a natureza complicada dos trabalhos são evidentes nas reflexões dos alunos, onde estes exprimiram sentimentos contraditórios e discutiram diferentes desafios e vitórias. Uma das vitórias mais mencionadas foi o fato de terem tido a oportunidade de se envolverem num projeto real com um impacto palpável num grupo de aprendentes. Uma das alunas escreveu na sua reflexão:

Em resumo, frequentar este curso consistiu numa experiência original e surpreendente para mim. Eu tive muitos altos e baixos mas lidei com os problemas o melhor que podia, e agora não tenho mais nada com que me preocupar. Este curso ajudou-me não só a compreender melhor o processo de desenho educacional, mas também me ensinou lições de vida importantes, uma das quais é, "espera o inesperado". Finalmente, aprender a trabalhar em equipe é chave no mundo do trabalho (Reflexão de uma aluna partilhada em dezembro de 2005; tradução nossa).

\section{Sugestões para o desenvolvimento de cursos a distância}

$\mathrm{O}$ estudo de caso descrito neste artigo permite-nos refletir sobre algumas das estratégias que resultaram da utilização dos modelos de aprendizagem experiencial e colaborativa num curso a distância. Espera-se que os leitores possam ver este caso como uma oportunidade de refletir e discutir sobre a aplicação destas estratégias aos seus próprios contextos de prática $e$ grupos de aprendentes. Aqui ficam algumas sugestões para o desenvolvimento de cursos a distância usando o enquadramento teórico e as práticas seguidas nestes estudos. É importante:

- conhecer bem os alunos inscritos no curso para que as atividades de aprendizagem coincidam com os seus interesses e vão ao encontro de suas expectativas como aprendentes;

- adicionar uma dimensão da realidade às atividades de aprendizagem;

- apoiar e incentivar o trabalho de grupo virtual, com conselhos práticos, estratégias para comunicar a distância e frequentes e variadas oportunidades de interação entre os membros da equipe; 
- oferecer aos alunos não só uma oportunidade de terminar o curso com sucesso, mas igualmente a satisfação de contribuir para a resolução de um problema educacional através da aplicação dos seus conhecimentos e competências em desenho educacional.

Através do emprego de uma pedagogia de e-learning que preenche o vácuo entre o ensino-apren- dizagem descontextualizado e a experiência prática e aplicada, os docentes podem educar indivíduos capazes de enfrentar novos desafios para além da sala de aula virtual. Este artigo tenta de forma humilde contribuir para uma reflexão que potencie um uso mais alargado de aprendizagem experiencial $e$ colaborativa na Educação a Distância. 


\section{Referências bibliográficas:}

CORREIA, A.P.; DAVIS, N. Intersecting communities of practice in distance education. Distance Education. Australia, v. 29, n. 3, p. 289-306, 2008.

CORREIA, A.P.; DAVIS, N.E. The design of collaboration in the virtual classroom. In: SIMONSON, M. (Ed.). 30th Annual Proceedings of Selected Papers on the Practice of Educational Communications and Technology. Anaheim, CA, USA, 2007. p. 84-87.

CORREIA, A.P. (2008). Moving from theory to realworld Experiences in an e-learning community. Innovate, USA, v. 4, n. 4. Disponível em: <http:// www.innovateonline.info/index.php?view $=$ article\&id $=495>$. Acesso em: 12 maio 2008 .

HARASIM, L. Shift happens: online education as a new paradigm in learning. The Internet and Higher Education, USA, v. 3, n. 1-2, p. 41-61, 2000.

KOLB, D. Experiential learning: experience as the source of learning and development New Jersey: Prentice-Hall, 1984.

KOLB, A.; KOLB, D. Learning styles and learning spaces: enhancing experiential learning in higher education. Academy of Management Learning \& Education, v. 4, n. 2, p. 193-212, 2005.

LEVAN, S. K. Travail collaboratif sur internet: concepts, méthods et pratiques des plateaux project. Paris: Vuilbert, 2004.

MCLOUGHLIN, C.; LUCA, J. A learner-centered approach to developing team skills through web-based learning and assessment. British Journal of Educational Technology, v. 33, n. 5, p. 571-582, 2002.

MEIRINHOS, M. Desenvolvimento professional docente em ambientes colaborativos de aprendizagem a distância: estudo de caso no âmbito da formação contínua. 2006. Tese (Doutorado) - Universidade do Minho, Braga, 2006.
MURPHY, E. Recognising and promoting collaboration in an online asynchronous discussion. British Journal of Educational Technology, v. 35, n.4, p. 421-431, 2004.

SMITH, B. L.; MACGREGOR, J. T. What Is Collaborative Learning? National Center on Postsecondary Teaching, Learning, and Assessment at Pennsylvania State University, 1992. Disponível em: $<$ http://learningcommons.evergreen.edu/pdf/collab. pdf $>$. Acesso em: 11 set. 2008. 